\title{
Paper Interference Aware Routing Game for Cognitive Radio Ad-hoc Networks
}

\author{
Soodeh Amiri-Doomari, Ghasem Mirjalily, and Jamshid Abouei \\ Department of Electrical Engineering, Yazd University, Yazd, Iran
}

https://doi.org/10.26636/jtit.2018.110817

\begin{abstract}
Cognitive radio is a new communication paradigm that is able to solve the problem of spectrum scarcity in wireless networks. In this paper, interference aware routing game, (IRG), is proposed that connects the flow initiators to the destinations. A network formation game among secondary users (SUs) is formulated in which each secondary user aims to maximize its utility, while it reduces the aggregate interference on the primary users (PUs) and the end-to-end delay. In order to reduce the end-to-end delay and the accumulated interference, the IRG algorithm selects upstream neighbors in a view point of the sender. To model the interference between SUs, IRG uses the signal-to-interference-plus noise (SINR) model. The effectiveness of the proposed algorithm is validated by evaluating the aggregate interference from SUs to the PUs and end-to-end delay. A comprehensive numerical evaluation is performed, which shows that the performance of the proposed algorithm is significantly better than the Interference Aware Routing (IAR) using network formation game in cognitive radio mesh networks.
\end{abstract}

Keywords-aggregate interference, end-to-end delay, routing game, network formation game.

\section{Introduction}

Due to the ability of cognitive radio (CR) to solve the problem of spectrum scarcity, spectrum congestion and underutilization, Cognitive Radio Networks (CRNs) have been recognized as an outstanding technology [1]. Recently, researchers consider lower layers' challenges such as spectrum sensing, sharing, and spectrum mobility in infrastructure-based networks that use a base-station for considering the spectrum information [2]-[4]. Cognitive Radio Ad-Hoc Networks (CRAHNs) as a new class of CRNs without any central entity [5] have been considered recently from different aspects including spectrum sensing, spectrum mobility and the routing issue in the network layer of CRAHNs [6]-[9]. As demonstrated in [10], routing challenges in CRAHNs are classified into three main categories: channel-based [5]-[9], host-based [4], [11], and network-based [7], [12], [13] routing.

Channel-based challenges are related to the operating environment, such as channel availability and diversity. Authors in [5] present a geographical routing algorithm that addresses three main goals: PUs receiver protection from SU interference, joint spectrum and route selection, and provisioning of different routing modes. In the proposed scheme, each SU calculates its overlapping transmission range with the PUs transmitters' coverage to minimize the probability of PUs receivers on that area.

A spectrum-tree based on-demand routing protocol (STODRP) for CRAHNs has been proposed in [6]. It simplifies cooperation between spectrum decision and route selection by establishing a spectrum tree in each frequency band. Since this algorithm uses control packets, the system's overhead is significantly high.

Authors in [7] consider route diversity effects on the actual cost of the route and suggest an optimal routing metric for CRAHNs. The presented routing algorithm focuses on the end-to-end delay for delay sensitive applications.

A geographical routing algorithm for mobile SUs has been proposed in [8] to minimize the interference from SUs imposed on PUs. The proposed scheme jointly undertakes the path and channel assignments to avoid the PU's footprint. In [9] the geometrical approach to improve the spectrum utilization is used. This work takes into account three main factors: SUs' interference on PUs, SU network reliability, and computing Quality of Service (QoS) in both SU and PU networks. For minimizing the SUs' interference on the PUs, the routing scheme calculates the maximum transmission range based on the transmission power and the location of SUs and PUs.

Host-based challenges are related to the SUs such as mobility and minimizing the channel switching delay or back off delay.

A route switching game to address spectrum mobility and route switching issues in CRAHNs has been proposed in [11]. The cost of data flow is modeled as routing and switching costs. Routing costs correspond to the end-toend delay and amount of energy consumption for relaying. Switching costs consider switching delay, back off delay and amount of energy consumption used for channel sensing and establishing new connections.

Network based challenges considers a tradeoff between the number of hop counts and other performance metrics such as interference, energy consumption and route robustness.

Article [12] proposes a new routing metric called cognitive transport throughput to capture the potential relay gain over next hop. The proposed scheme is based on the local channel usage statistics and selects the best relay node with the highest forwarding gain. In addition, a heuristic algo- 
rithm is proposed to decrease the searching complexity of the optimal selection of channels and relays.

The routing algorithm in [13] is aware of the degree of connectivity of possible paths towards the destination. In the proposed scheme, the authors present a new CR metric based on the path stability and availability over time.

The authors in [14] develop a routing strategy for CR mesh networks based on the network formation game. This scheme by avoiding PU's region minimizes the aggregate interference from SUs to the PUs. It does not consider the geographical location of the destination in finding the routes.

The authors in [15] present a distributed dynamic routing protocol in multi-hop CR-based on the non-cooperative game theory where SUs minimize their interferences imposed on PUs.

In [16], the authors introduce the route robustness for the path selection in multi-hop CR networks. The algorithm selects some routes from a robust route set and specifies the spectrum of the selected routes in a way that the throughput of the system is maximized. The proposed strategy is not feasible in CRAHNs as it needs a global knowledge about the network's topology.

A spectrum and energy aware routing algorithms for CRAHNs based on the dynamic source routing has been proposed in [17]. Although, the proposed scheme can balance energy consumption and is able to reduce the routing overhead, it does not consider the problem of aggregate interference from SUs to PUs.

\subsection{Contributions and Paper Organization}

A main obstacle of getting a high performance of routing algorithm is the interferences [18], [19]. This is a major factor in determining the boundaries for the spectrum reuse. Network throughput has a direct relationship with the interference among links. Due to the negative impact of interference, the QoS of the network will be changed with the change of the routing patterns. Estimating the interference in a CRAHN is not an ordinary task. Therefore, proposing an efficient interference-aware routing algorithm that considers the interference measures to reduce its effects on each PU is a challenging task. Toward this goal, we define the PU's footprint as an area that no SU allowed to be turned on. According to the mentioned problem, we propose the interference aware routing algorithm for CRAHNs based on the network formation game (IRG).

In the proposed algorithm, a game theory model is used to connect the flow initiators directly or through SUs to the intended destinations optimally. The designed protocol is distributed, that avoids the problems of centralized algorithms. In contrast with the [14], proposed method selects an upstream neighbor that is close to the destination and out of the PU's region by geographical routing. In this way, we introduce the relay coefficient value (RCV) metric.

$\mathrm{RCV}$ helps to reduce the end-to-end delay and decreases the interference from SUs imposed on the PUs. The most important difference between proposed method and [14] is that this work considers the amount of interference between SUs and moreover it focuses on the physical interference model as in [20]. Another advantage of the proposed algorithm compared with [14] is the load balancing technique that leads to decrease the network congestion and also decreases the amount of aggregate interference on the PUs. Simulation results in four different scenarios show that the proposed protocol achieves a superior performance with reducing the normalized aggregate interference and the number of hop counts compared to [14].

The rest of this paper is organized as follows. Section 2 contains the system model description and assumptions. Section 3 introduces the proposed algorithm. In Section 4, the network formation game is presented. In Section 5, the performance of the proposed algorithm is evaluated. Finally, in Section 6, an overview of the results and some conclusion remarks are presented. For convenience, a list of key mathematical symbols used in this paper is provided in Table 1.

Table 1

System parameters

\begin{tabular}{|c|c|}
\hline Symbol & Definition \\
\hline \hline $\mathbf{G}$ & Network graph \\
\hline $\mathbf{V}$ & Set of SU nodes \\
\hline $\mathbf{E}$ & Set of edges (links) created between nodes \\
\hline$l(i, j)$ & Link from node $i$ to node $j$ \\
\hline$R_{I}$ & Interference range \\
\hline$R_{T}$ & Transmission range \\
\hline$C_{i j}$ & Capacity of link $l(i, j)$ \\
\hline$n u m_{i}$ & Number of neighbor nodes of node $i$ \\
\hline$t_{i}$ & $\begin{array}{c}\text { Amount of generated traffic } \\
\text { by node } i \text { in a unit of time }\end{array}$ \\
\hline$p(i)$ & Transmission power of node $i$ \\
\hline$t_{i, j}$ & $\begin{array}{c}\text { Amount of traffic from node } i \\
\text { to node } j \text { in a unit of time }\end{array}$ \\
\hline$P\left(f_{k}\right)$ & Determined path for flow $f_{k}$ \\
\hline$d_{i, j}(t)$ & Euclidean distance between nodes $i$ and $j$ \\
\hline$W$ & Bandwidth \\
\hline
\end{tabular}

\section{System Model and Assumptions}

\subsection{Network Model and Assumptions}

In this work, we consider a multi-hop CRAHN consisting of $M$ stationary and location-aware SU nodes, denoted by $\{1,2, \ldots, M\}$ and there are $K$ stationary PUs indexed by $\{1,2, \ldots, K\}$. All nodes are distributed randomly through- 
out an $\mathrm{A} \times \mathrm{A}$ square area. We assume that there are $N$ data flows $f_{k}=\left(n_{f_{k}}, D_{f_{k}}\right)$, where $k=1,2, \ldots, N$ corresponds to $k$-th data flow, $n_{f_{k}}$ and $D_{f_{k}}$ are $k$-th flow initiator and $k$-th destination, respectively. Any flow initiator knows the location information of intended destination. SUs can acquire their own location information using the Global Positioning System (GPS) or other available localization services. Figure 1 shows proposed game strategy. There are six SUs and one PU deployed randomly in the area. The big circle represents the coverage area of PU. $S$ and $D$ represent the source and destination nodes, respectively. $v_{1}$ and $v_{4}$ could not participate in routing process because $v_{1}$ is in the coverage area of PU and $v_{4}$ is a downstream neighbor of $S$. Since node $v_{3}$ is further to the primary user compared to node $v_{2}$, node $S$ selects $v_{3}$ as a next hop, because further node creates less interference on PU. In this work, the network is

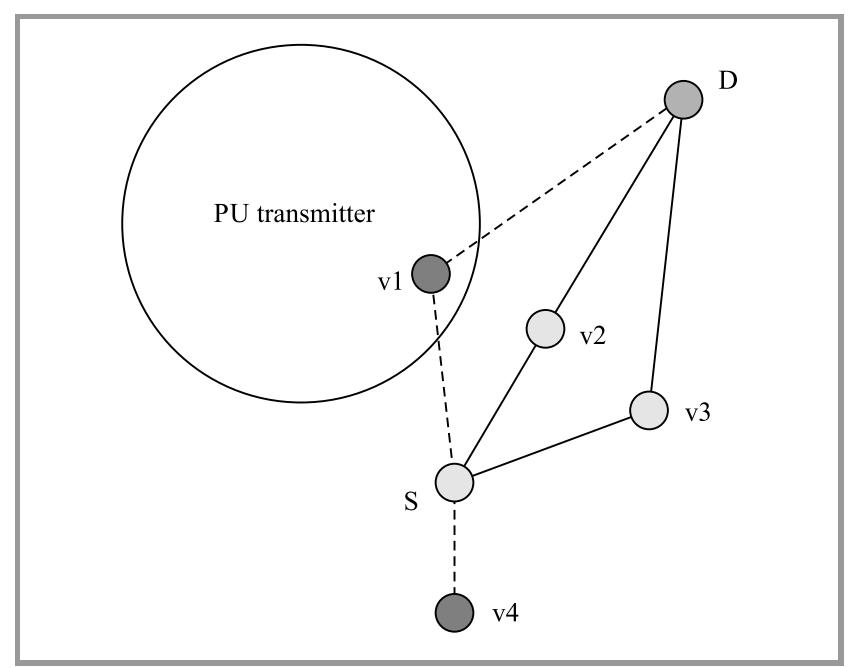

Fig. 1. Implementation of game strategy for $M=6$ SUs distributed in the presence of the PU.

modelled by a directed graph $\mathbf{G}=(\mathbf{V}, \mathbf{E})$, where $\mathbf{V}$ represents $M$ SU nodes and $\mathbf{E}$ denotes the set of links. We define a path for flow $f_{k}$ as $P\left(f_{k}\right)=\left\{v_{1}^{k}, v_{2}^{k}, \ldots, v_{h-1}^{k}, v_{h}^{k}\right\}$, where $h$ is the hop count and the nodes $v_{1}^{k}$ and $v_{h}^{k}$ correspond to the source and destination nodes, respectively.

\subsection{Interference Model}

Similar to [20], we consider two interference models: protocol model and physical model.

In protocol model, a transmission from node $i$ to $j \underset{i, j}{\forall} \in$ $\{1,2, \ldots, M\}$ is successful if both of the following conditions are satisfied:

- $d_{i, j}(t)<R_{T}$, where $R_{T}$ is the transmission range of SUs;

- any node $k$ with $d_{k, j}(t)<R_{I}$ is not transmitting, where $R_{I}$ is the interference range. On the other hand, a node may not send and receive at the same time and it cannot transmit to more than one node at the same time.
In physical model, suppose that node $i$ wants to transmit to node $j$. The transmission is successful if:

$$
\operatorname{SINR}(i, j)=\frac{g(i, j) p(i)}{\eta W+\Sigma_{k \in \mathbf{V}, k \neq i, j} g(k, j) p(k)} \geq \sigma,
$$

where $\eta$ is the ambient Gaussian noise density, $g(i, j)=$ $\left[d_{i, j}(t)\right]^{-\alpha}$ is the propagation loss from node $i$ to node $j$ where $\alpha$ is the path loss exponent, $p(i)$ is the transmission power of node $i$ and $d_{i, j}(t)$ is the Euclidean distance between nodes $i$ and $j$. A link $l(i, j)$ is available if the following conditions are satisfied:

$$
\begin{aligned}
& \text { - } \operatorname{SINR}(i, j) \geq \sigma, \\
& \text { - } d_{i, P U}(t)>R_{I} \text { and } d_{j, P U}(t)>R_{I},
\end{aligned}
$$

where $d_{i, P U}(t)$ and $d_{j, P U}(t)$ are the Euclidean distances between node $i$ and $P U$, and node $j$ and $P U$, respectively. The binary variable $\beta_{i j}$ indicates the existence of a potential directed link from node $i$ to $j$ :

$$
\beta_{i j} \triangleq \begin{cases}1 & , \text { if there exist a potential link } l(i, j) \\ 0 & , \text { otherwise }\end{cases}
$$

According to the Shannon's formula [21], the capacity of link $l(i, j)$ is defined as:

$$
C_{i j}=W \log _{2}(1+\operatorname{SINR}(i, j))
$$

The amount of traffic on node $i$ must satisfy the following conditions:

$$
\begin{gathered}
t_{i}+\sum_{j \in \mathbf{V}} \beta_{j i} \times t_{j, i}-\sum_{j \in \mathbf{V}} \beta_{i j} \times t_{i, j}=0 \\
t_{i, j} \leq C_{i j}
\end{gathered}
$$

Equations (4) and (5) are the flow conservation constraints. In Eq. (4) outgoing flow should be equal to the sum of incoming flow and generated traffic. In Eq. (5) flow on each link cannot be bigger than its capacity.

Additionally, for minimizing the aggregate interference from SUs to the PU, primary user has a footprint where no SU is allowed to be turn on. When the SUs are outside the PU's footprint, they can utilize the cognitive functionalities to access the licensed spectrum. It is clearly predictable that with an increase in the number of secondary users $M$, the amount of aggregate interference is increased. The total interference to the primary user for a path $P\left(f_{k}\right)$ between $v_{1}^{k}$ and $v_{h}^{k}$ is given by:

$$
I(P)=\sum_{i=1}^{h} p(i) L_{i}
$$

where $p(i)$ is the transmission power of node $v_{i}^{k}, L_{i}=d_{i, P U}^{-\alpha}$ is the propagation loss from SU to the PU where $d_{i, P U}$ is the Euclidean distance between node $v_{i}^{k}$ and PU.

Figure 2 demonstrates the IRG interference model. There are a PU and three SUs deployed randomly in the area. The solid lines represent the channel interference from SUs to the PU, and the dotted lines show the channel interference from a SU to another SU. 


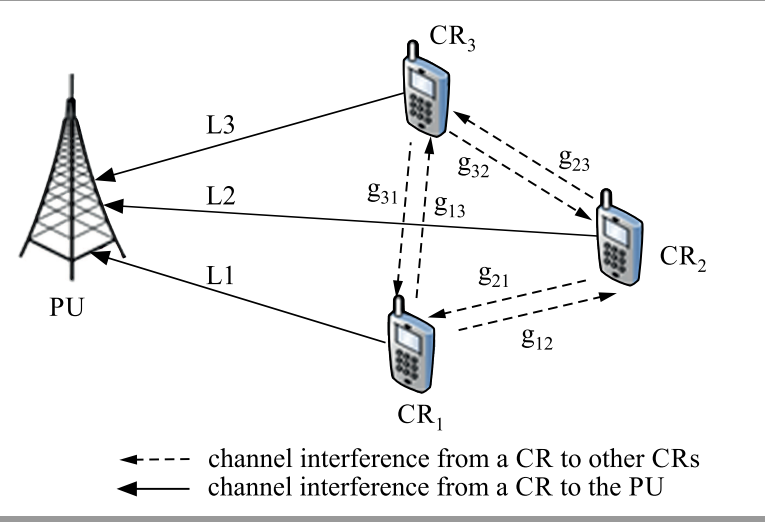

Fig. 2. System interference model when there are one PU and three SUs.

\section{Interference Aware Routing Game for CR Ad-hoc Networks}

Network formation game is a field of game theory that offers a suite of tools that may be used effectively in modeling the interaction among SU nodes in ad hoc networks to improve their payoffs [14], [22]. Game $g=\{\mathbf{V}, \mathbf{S}, U(a)\}$ has three main components: players, game strategy, and utility function. The proposed game components are defined as:

Definition 1. V shows a set of players or decision makers. Each player evaluates the resulting outcome through a payoff or "utility" function representing its objective. Interaction between the players is represented by the influence that each player has on the resulting outcome after other players have selected their actions. In the proposed algorithm, players are SUs, which establish a connection with their neighbors to route the traffic by focusing on minimizing the aggregate interference on PUs.

Definition 2. $\mathbf{S}=\left\{s_{1} \times s_{2} \times \cdots \times s_{M}\right\}$ is the strategy space. In fact, strategy is a decision to forward packets or not. SU nodes choose a strategy from their strategy space in response to other players' strategies. According to the sender (i.e. $S$ ) and destination (i.e. $D$ ) locations, only upstream neighbors (i.e. $R$ ), can forward packets. Choosing upstream neighbors, in the view point of $S$, leads to reduce the number of transmissions, end-to-end delay and the energy consumption. To specify the upstream neighbors, we define the $R C V(S, R, D)$ as:

$$
R C V(S, R, D)=d_{S, D}(t)-d_{R, D}(t),
$$

where $d_{S, D}(t)$ and $d_{R, D}(t)$ show the Euclidean distance between source $S$ and destination $D$ and between relay $R$ and destination $D$, respectively. We assume that each node knows the location information of its neighbors. In this case, each node sets its location information on the hello packet and broadcasts it.

To prevent the creation of loops in the network graph, it is necessary that if player $j$ is connected to $i$ already, player $i$ cannot choose player $j$ as its strategy. More precisely, if a link $l(j, i) \in \mathbf{E}$, then link $l(i, j) \notin \mathbf{E}$. To satisfy the above conditions, game strategy is defined as:

$$
s_{i}=\left\{l(i, j) \in \mathbf{E} \mid j \in \mathbf{V} \backslash\{i\} \cup \lambda_{i}, R C V(i, j, D)>0\right\},
$$

where $\lambda_{i}$ is the set of nodes from which node $i$ is accepted a link $l(j, i)$, i.e.

$$
\lambda_{i}=\{j \in \mathbf{V} \backslash\{i\} \mid l(j, i) \in \mathbf{E}\} .
$$

Definition 3. In the presented network formation game, each player has a utility that basically contributes every player in the network to improve its payoff by choosing a less congested node that is not only far from PU, but also near the destination. In fact, outcomes are determined by the particular strategy chosen by player $i, s_{i}$, and the strategies chosen by all of the other players in the game, $\mathbf{S}_{-i}$. The utility function in [14] is composed of barrier functions, interference temperature, link capacity and the amount of flow. By inspiration, we define the utility function of player $i$ when it selects neighbor $j$ as follows:

$$
U(i, j)=\beta_{i j} \frac{R C V(i, j, D) \times C_{i j}}{\bar{E}_{j} t_{i, j} T_{I j}},
$$

where $\bar{E}_{j}=\frac{1}{n^{\prime} m_{j}+1}\left(E_{j}+\sum_{i=1}^{\text {num }_{j}} E_{i}\right)$ is the average current traffic load on node $j$ and its neighbors in bits per seconds, num $_{j}$ is the number of neighbor nodes of node $j, T_{I j}=$ $\frac{P_{I j}}{k_{\mathrm{B}} W}$ is the interference temperature of node $j, P_{I j}$ is the interference power in watts imposed by node $j$ and $k_{\mathrm{B}}$ is the Boltzman's constant in $\mathrm{J} / \mathrm{K}$ and $E_{i}$ is the total traffic on node $i . \bar{E}_{j}$ caused to balance the load in the network and avoids the network congestion. If the area around the $\mathrm{PU}$ is quieter, the amount of aggregate interference will be decreased significantly.

\section{Proposed Network Formation Algorithm}

In this section, we proposed the network formation algorithm in details and some preliminary concepts are presented. When all SUs except $i$ keep their own strategies $\mathbf{S}_{-i}=\left\{s_{1}, \ldots, s_{i-1}, s_{i+1}, \ldots, s_{M}\right\}$, the network graph is defined as $\mathbf{G}_{s_{i}, \mathbf{S}_{-i}}$. All players to improve their utilities choose appropriate strategies. For instance, player $i$ selects strategy $s_{i}=l(i, j) \in \mathbf{S}_{i}$, player $j$ may refuse to accept this connection if it reduces the utility of node $j$.

There are several approaches for the network formation game that are classified into two categories: myopic [14], [22] and far sighted [23]. The main difference between these two approaches is that in the myopic, the players employ their strategies based on the current state of the network. In other words, each player does not consider the future evolution of the network when it wants to maximize its payoff. However, in the far sighted algorithm, players adapt their strategies by predicting future strategies 
of other players. For both types, well-known concepts of non-cooperative game theory can be used. The presented network formation algorithm which is summarized in Algorithm 1 is based on the myopic non-cooperative game. Time axis is divided into slots with the fixed duration $\tau$, each time slot is a round of the game. In each round, flow initiators generate a random number between 0 and 1 , which indicates its priority. Each SU node utilizes its own strategy, $s_{i}^{*} \in \mathbf{S}_{\mathbf{i}}$, to calculate its current utility by Eq. (10). If $U\left(\mathbf{G}_{s_{i}^{*}, \mathbf{S}_{-i}}\right)>U\left(\mathbf{G}_{s_{i}, \mathbf{S}_{-i}}\right)$ strategy $s_{i}^{*} \in \mathbf{S}_{\mathbf{i}}$ is a best response for a player $i \in \mathbf{V}$. To find the best response, players employ pairwise negotiations with their upstream neighbors. Assume that player $i$ wants to form a new link with $j$. Adding a new link increases the amount of load on $j$. Therefore a link formation affects on utility of both nodes $i$ and $j$. Hence, both nodes should consider the effect of link formation on their utilities before doing the actual formation. In this case, we consider the pairwise stability.

Definition 4. (pairwise stability): Under both following conditions, a network $\mathbf{G}$ is pairwise stable:

$$
\begin{aligned}
& \text { 1. } l(i, j) \in \mathbf{E}, U_{i}(\mathbf{G}) \geq U_{i}(\mathbf{G}-l(i, j)) \text { and } U_{j}(\mathbf{G}) \geq \\
& \geq U_{j}(\mathbf{G}-l(i, j))
\end{aligned}
$$

2. $l(i, j) \notin \mathbf{E}$ if $U_{i}(\mathbf{G}+l(i, j)) \geq U_{i}(\mathbf{G})$ then $U_{j}(\mathbf{G}+$ $+l(i, j))<U_{j}(\mathbf{G})$.

In other words, by removing a link, amount of utility of both players $i$ and $j$ should be increased and also forming a new link should have a positive effect on both $i$ and $j$ utilities [24].

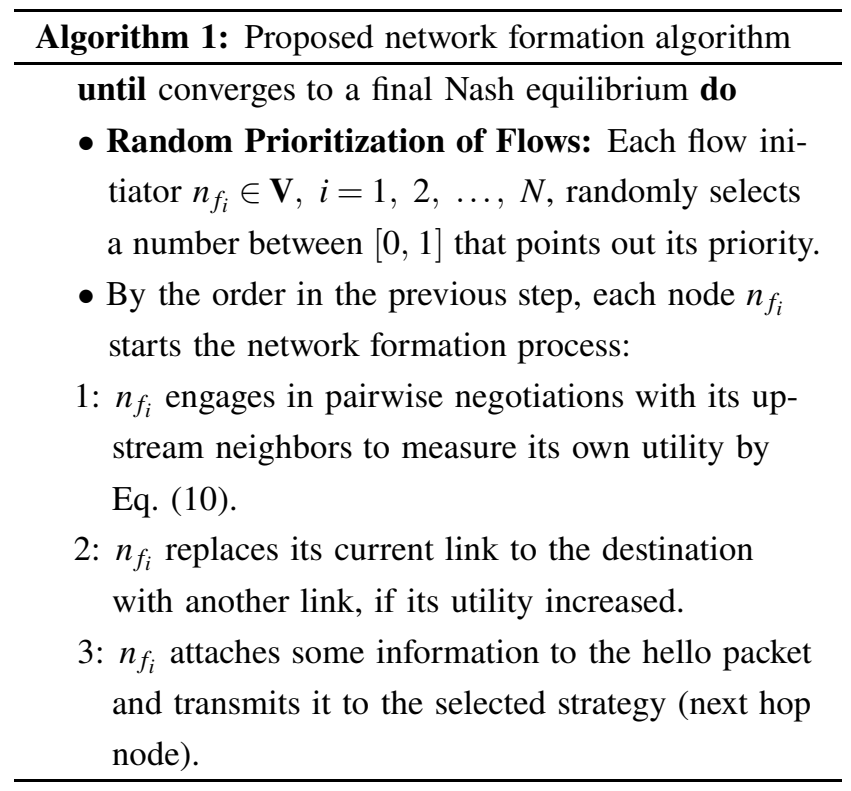

Based on the pairwise stability, flow initiators choose their best responses and leave the game until the next round, while its selected strategies enter the game. When the proposed network formation algorithm converges to the Nash equilibrium (NE), it reaches to a network where no player can change its strategy (current link).
Definition 5. Players cannot improve their own utilities by unilaterally changing the strategy at the equilibrium [14]. Therefore, a Nash graph is formed where the links chosen by each user are the best strategy.

In this model, player $i \in \mathbf{V}$ can choose its strategy $s_{i}=$ $l(i, j) \in \mathbf{S}_{\mathbf{i}}$ to improve its utility, while another player $j$ can decline the $i$ 's request, if it leads to the utility reduction of node $j$. When no node in the network could change its payoff, the NE is achieved. In the proposed method, hello packets are sent until the network converges to the NE. The fields of one hello packet are shown in Table 2. When

Table 2

Fields of the hello packet

\begin{tabular}{|c|c|}
\hline Fields & Descriptions \\
\hline \hline Des-Pos & Position of the destination node \\
\hline $\begin{array}{c}\text { Sender- } \\
\text { Pos }\end{array}$ & Position of sender \\
\hline$\overline{E_{j}}$ & Average traffic load on sender node $j$ \\
\hline TTL & Limitation of hop-length of the path \\
\hline
\end{tabular}

node $j$ is selected as the next hop, it attaches the following information to the hello packet:

- its own location information,

- average amount of its traffic load represented by $\bar{E}_{j}$,

- value of Time To Live (TTL) parameter.

The purpose of the TTL is to limit the number of hop counts in the selected path. After receiving a hello packet, a node checks the value of TTL. If it is zero and the node is not the destination, the node drops the hello packet. Otherwise, the node decreases the value of TTL by one.

Lemma 1: User $i$ aims to optimize its own utility along its path to the destination rather than to have a control over the selection of other nodes.

Proof: User $i$ only has control ability over its neighbor node, while other links are not controlled by node $i$. This means that node $i$ cannot choose the full path directly and only can increase its payoff. To generate a multi-hop connection from flow initiator $n_{f_{i}}$ to destination $D_{f_{i}}$, a selected node $i$ needs to find a node in its neighborhood to connect as a next hop. In fact, SU $i$ needs to choose a path that result in an optimal payoff. However, node $i$ cannot choose the full path as together and it is not important the choice of other nodes. The path utility of user $i$ can be expressed as a sum of utilities of all the nodes in the path. Denoting $U_{i}^{*}$ as the optimal path utility of node $i$, we have:

$$
U_{i}^{*}=\max \left(\sum_{n=i}^{h} U(n, n+1)\right)
$$

where $h$ is the hop count of path. Since each node in the path $\left(n_{f_{i}}, D_{f_{i}}\right)$ maximizes its payoff, the path utility in 
Eq. (11) can be rewritten as:

$$
\begin{aligned}
U_{i}^{*}=\max (U(i, i+1))+ & \max \left(\sum_{n=i+1}^{h} U(n, n+1)\right)= \\
& =\max (U(i, i+1))+U_{i+1}^{*} .
\end{aligned}
$$

\section{Simulation Results}

The performance of the proposed algorithm is evaluated and compared in different scenarios with the IAR algorithm [14] in terms of the end-to-end delay and the aggregate interference. For the scenarios under simulation, we show the effect of number of SU nodes, and the distance between flow initiators and destination, on the aforementioned performance metrics, and show the superiority of our proposed algorithm compared with the traditional IAR algorithm.

\subsection{Simulation Setup}

We consider a CRAHN in which $M$ SU nodes are randomly distributed with the uniform distribution inside a square area with the size $400 \times 400 \mathrm{~m}^{2} . M$ is selected from the set $\{50,70,90,110\}$. We assume that there are $K$ PUs in determined locations. There are five flow initiators that send their data to the intended destinations through the specified path $P$. For each data set, the location and traffic volume of each flow initiators (except $n_{f_{1}}$ ) are randomly selected. We assume that $R_{T}=90 \mathrm{~m}$ and $R_{I}=180 \mathrm{~m}$.

In the simulations, we consider IEEE $802.11 \mathrm{~g}$ standard. According to this standard, the physical layer is based on the Orthogonal Frequency Division Multiplexing (OFDM). Here, we consider the transmission rates of $6,9,12,18$, 24, 36 and 48 Mbps. In Eq. (1), $\eta$ is the ambient Gaussian noise density, which is $k_{\mathrm{B}} T$, where $T$ is ambient temperature and $k_{\mathrm{B}}$ is Boltzmann constant. By considering $k_{\mathrm{B}}=1.38 \cdot 10^{-23} \mathrm{~J} / \mathrm{K}, T=300 \mathrm{~K}, \eta=414 \cdot 10^{-23}$ and $W=20 \mathrm{MHz}$.

In addition, each node computes its utility by Eq. (10). Each player to improve its utility saves the amount of its previous utility. To achieve the NE, players play in game while no SU can improve their utilities. We assume that each round of game is $20 \mathrm{~s}$.

In the simulations, the data packet has an exponential distribution with mean 50 bytes. Finally, we compute the average of each performance metric over some runs where the corresponding $95 \%$ confidence intervals are also reported. Figure 3 shows the convergence of the selected node when $M=110$. As seen from Fig. 3, the amount of the utility in some iterations (before the proposed algorithm reaches to the NE point) follows decrease/increase behaviors. It is a well-known fact that in each iteration, a selected node intends to choose the best partner to connect in order to improve its utility. Hence, the selected node may not change its partner in some iterations. Therefore, the amount of its utility changes until the game reaches a steady state or the NE point.

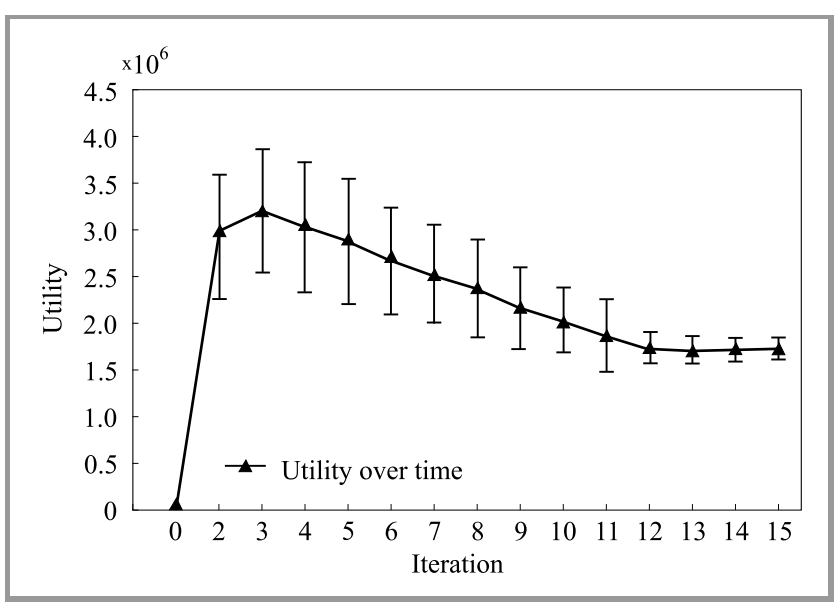

Fig. 3. Utility of a sample node over 15 iterations.

\subsection{Evaluation and Comparison}

First scenario: In this scenario, the number of SUs is selected from set $\{50,70,90,110\}$ and there is one stationary PU located in $(30,374)$. Figure 4 compares the normalized aggregate interference of the proposed algorithm with that of the IAR in [14] for the first flow versus the different number of SUs. The normalized aggregate interference is defined as the amount of the aggregate interference, Eq. (6), imposed on the PU divided to the maximum value. As seen from Fig. 4, the proposed scheme displays a lower interference imposed to the PU when compared to the presented

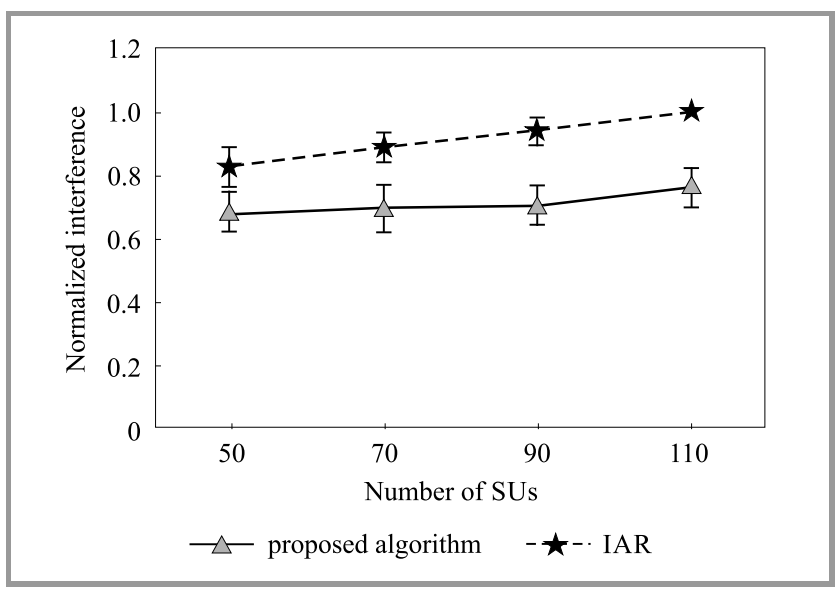

Fig. 4. Normalized interference versus different number of SUs in the proposed IRG and IAR algorithms.

algorithm in [14]. The minimum interference is achieved when the number of SUs is equal to 50 . This enhancement comes from the $\bar{E}_{j}$ used in the proposed utility function. In fact, $\bar{E}_{j}$ causes the algorithm keeps away from routes that are located in the congested network area. More precisely, if the density of the flow in the region near the PU is high, the amount of the aggregate interference that secondary users create on the PU is increased significantly.

Figure 5 compares the number of hop counts (or equivalently end-to-end delay) between flow initiator $n_{f_{1}}$ and destination $D$ versus different number of SUs for both 
algorithms. The interesting point from Fig. 5 is that in the proposed algorithm, RCV prevents packets to transmit to the downstream neighbors, thus, the end-to-end delay and the amount of energy consumption of the proposed scheme are reduced significantly when compared to the IAR algorithm.

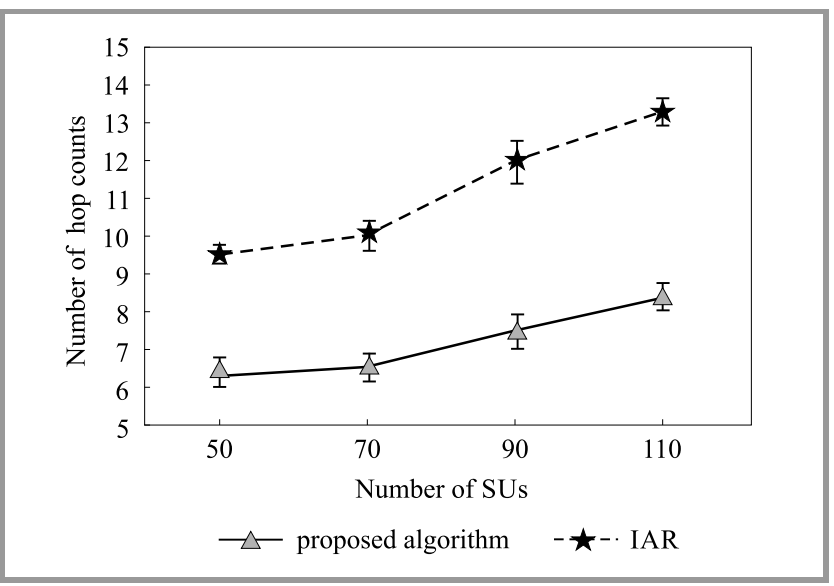

Fig. 5. Number of hop counts between $n_{f_{1}}$ and $D$ versus different number of SUs in the proposed IRG and IAR algorithms.

Second scenario: In this scenario, the number of SUs is fixed at $M=110$, we set $K=1$, PU is located in $(30,374)$, and the distance between flow initiator $n_{f_{1}}$ and $D$ is variable in the range of $[50,350]$. Figures 6 and 7 provide a fair comparison between our algorithm and the IAR scheme in terms of the normalized aggregate interference and the endto-end delay. Clearly, when the distance between $n_{f_{1}}$ and $D$ is low, the performances of both schemes are the same. The result comes from the fact that the route between source and destination will not include more nodes. However, by increasing the distance between the flow initiator and the destination, the number of hop counts is increased and the

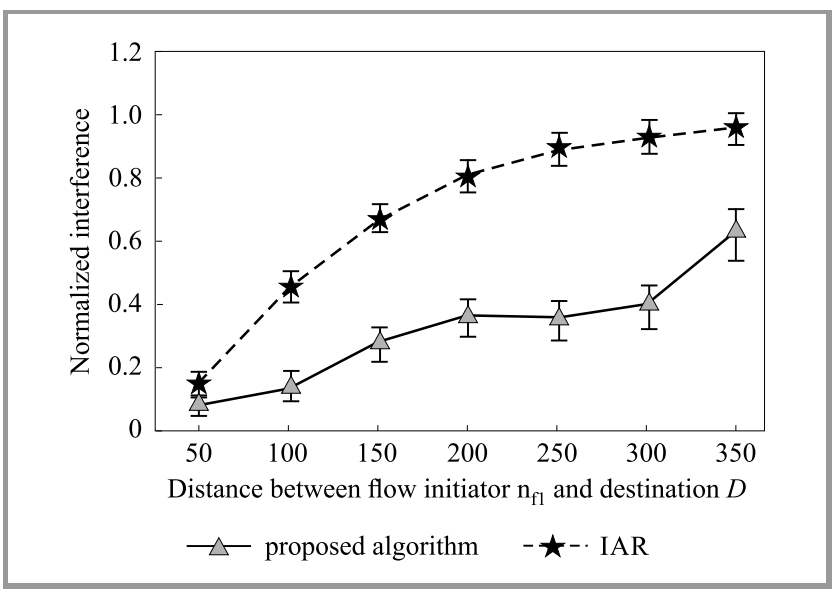

Fig. 6. Normalized interference versus different distances between $n_{f_{1}}$ and $D$ in the proposed IRG and IAR algorithms.

route consists of more SU nodes. Thus for both algorithms, the aggregate interference from more SUs which are transmitting in a unit of time is increased significantly.

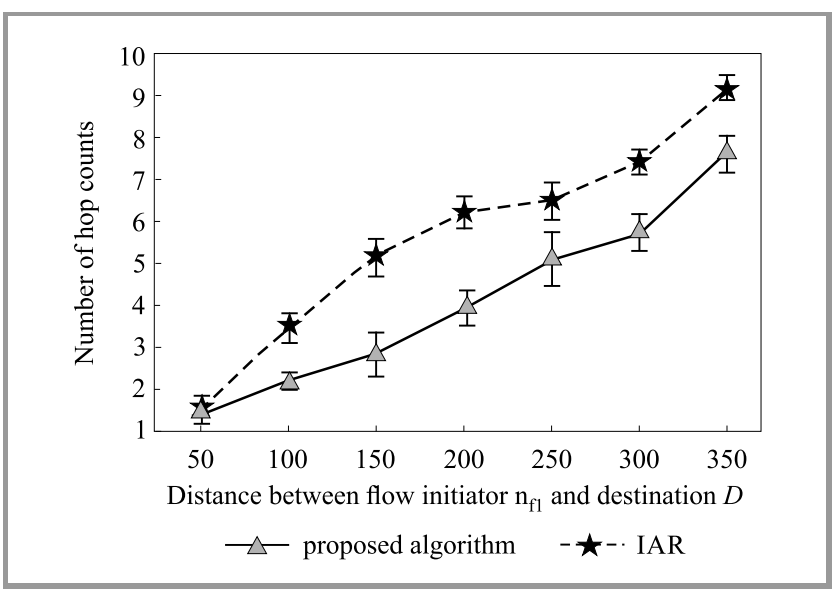

Fig. 7. Number of hop counts between $n_{f_{1}}$ and $D$ versus different distances between $n_{f_{1}}$ and $D$ in the proposed IRG and IAR algorithms.

Third scenario: In this scenario, we set $K=2$, and the number of SUs is changed over the range $\{50,70,90,110\}$. We follow the same performance metrics as in the first scenario to compare our proposed IRG scheme with that of

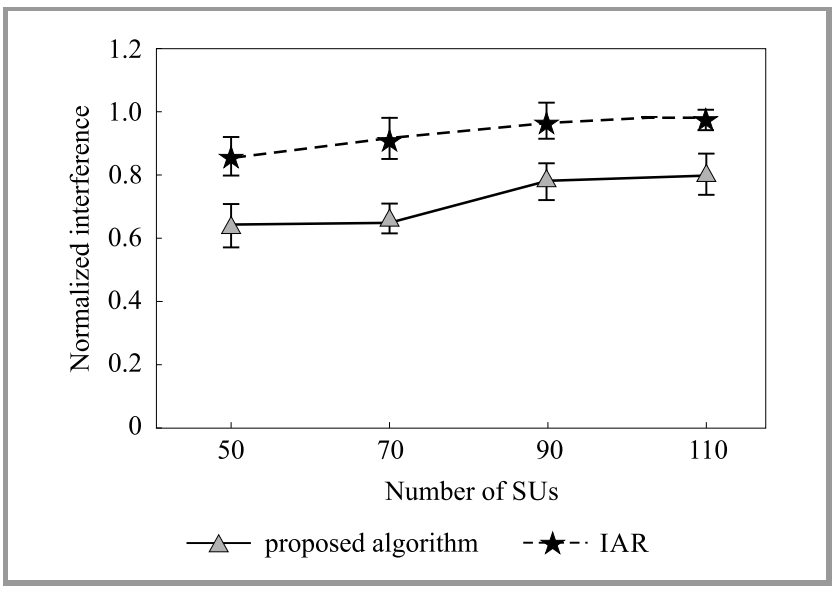

Fig. 8. Normalized interference when $K=2$ vs. different number of SUs in the proposed IRG and IAR algorithms.

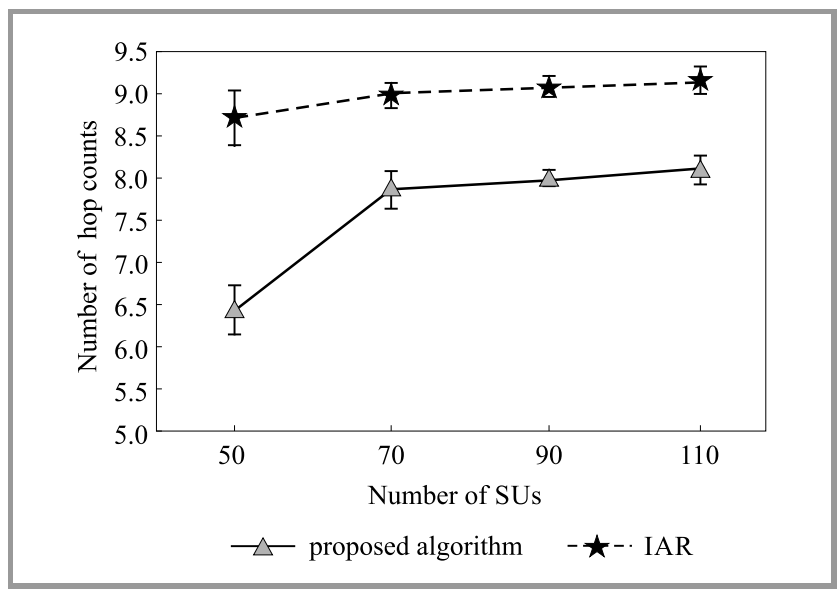

Fig. 9. Number of hop counts between $n_{f_{1}}$ and $D$ when $K=2$ vs. different number of SUs in the proposed IRG and IAR algorithms. 
the conventional IAR method when there are two PUs in determined locations $(140,187)$ and $(30,374)$. Similar to the first scenario, with an increase in the number of SUs, the amount of normalized interference imposed on both PUs is increased, and as a result, the number of hop counts grows, as respectively observed from Figs. 8 and 9.

Fourth scenario: To complete our simulation results, we evaluate the normalized aggregate interference and the end-to-end delay when physical inteference model is used and the link capacity is calculated by Eq. (3). Figures 10 and 11 show the results of the proposed algo-

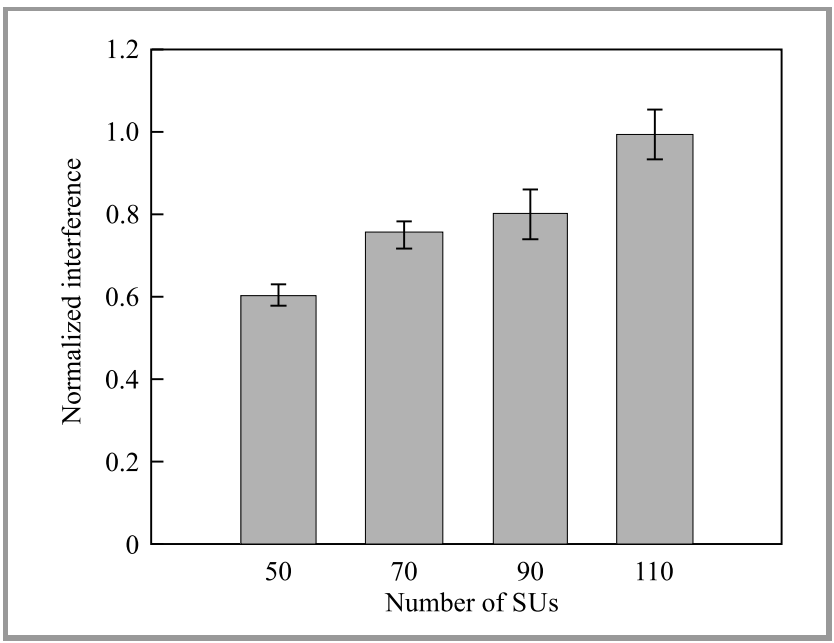

Fig. 10. Normalized interference given different number of secondary nodes in the proposed algorithm.

rithm in terms of the normalized interference and hop counts versus different number of SUs. The results are similar to the arguments as in Figs. 4 and 5. By increasing the number of SUs, the accumulated interference imposed on the PU and also the number of hop counts between the flow initiator $n_{f_{1}}$ and destination $D$ is increased significantly.

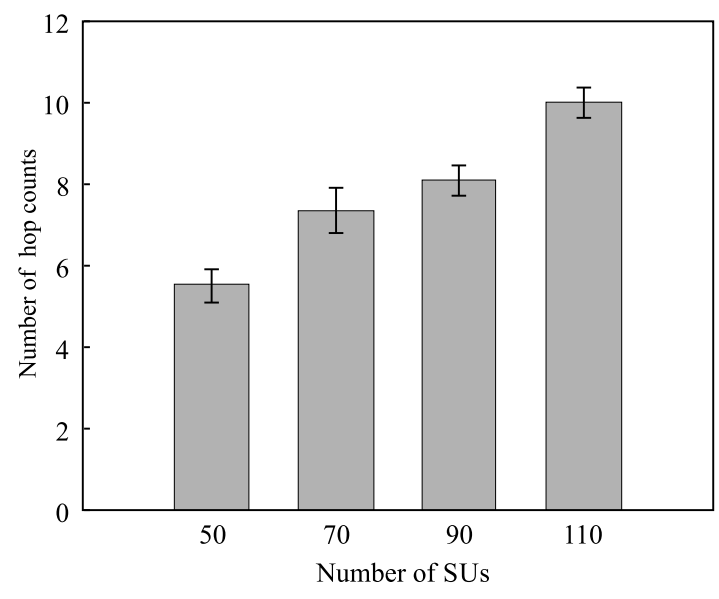

Fig. 11. Number of hop counts given different number of secondary nodes in the proposed algorithm.

\section{Conclusion}

In this paper, we used the game theory in CRAHNs to propose a new routing algorithm to control the interference and the number of hop counts. To this end, we formulated a network formation game among SUs and introduced a new utility function.

Using the proposed network formation algorithm, each SU can take a locally decision to optimize its utility by selecting a suitable strategy based on the myopic non-cooperative game. The proposed routing algorithm specifically mitigates the interference from SUs imposed on the PUs. Furthermore, to characterize the interference between SUs, we used the physical interference model. To select an appropriate neighbor, our game rule is to select an upstream neighbor in the view point of the sender nodes. We showed that the proposed algorithm avoids congested network zones and it forms at least one path from the flow initiators to the destinations. Simulation results showed that the proposed approach minimizes the aggregate interference and the number of hop counts between the flow initiator and the destination compared to the classical IAR [14] when the number of SUs is randomly selected over the set $\{50,70,90,110\}$.

\section{References}

[1] S. Haykin, Cognitive dynamic systems: perception-action cycle, radar and radio. Cambridge: Cambridge University Press, 2012 (ISBN: 978-0521114363).

[2] C. Cormio and K. R. Chowdhury, "A survey on MAC protocols for cognitive radio networks", Ad Hoc Networks, vol. 7, no. 7, pp. 1315-1329, 2009 (doi: 10.1016/j.adhoc.2009.01.002).

[3] K. B. Letaief and W. Zhang, "Cooperative communications for cognitive radio networks", Proceedings of the IEEE, vol. 97, no. 5, pp. 878-893, 2009 (doi: 10.1109/JPROC.2009.2015716).

[4] Z. Li, F. R. Yu, and M. Huang, "A distributed consensus-based cooperative spectrum-sensing scheme in cognitive radios", IEEE Trans. on Vehic. Technol., vol. 59, no. 1, pp. 383-393, 2010 (doi: 10.1109/TVT.2009.2031181).

[5] K. R. Chowdhury and I. F. Akyildiz, "CRP: A routing protocol for cognitive radio ad hoc networks", IEEE J. on Selec. Areas in Commun., vol. 29, no. 4, pp. 794-804, 2011 (doi: 10.1109/JSAC.2011.110411).

[6] G.-M. Zhu, I. F. Akyildiz, and G.-S. Kuo, "STOD-RP: A spectrumtree based on-demand routing protocol for multi-hop cognitive radio networks", in Proc. IEEE GLOBECOM Telecommun. Conf., pp. 1-5, New Orleans, LA, USA, 2008 (doi: 10.1109/GLOCOM.2008.ECP.592).

[7] M. Caleffi, I. F. Akyildiz, and L. Paura, "OPERA: Optimal routing metric for cognitive radio ad hoc networks", IEEE Trans. on Wireless Commun., vol. 11, no. 8, pp. 2884-2894, 2012 (doi: 10.1109/TWC.2012.061912.111479).

[8] K. R. Chowdhury and M. Felice, "Search: A routing protocol for mobile cognitive radio ad-hoc networks", Comp. Commun., vol. 32, no. 18, pp. 1983-1997, 2009 (doi: 10.1016/j.comcom.2009.06.011).

[9] M. Xie, W. Zhang, and K.-K. Wong, "A geometric approach to improve spectrum efficiency for cognitive relay networks", IEEE Trans. on Wireless Commun., vol. 9, no. 1, pp. 268-281, 2010 (doi: 10.1109/TWC.2010.01.090180).

[10] H. A. Al-Rawi and K.-L. A. Yau, "Routing in distributed cognitive radio networks: A survey", Wireless Person. Commun., vol. 69, no. 4, pp. 1983-2020, 2013 (doi: 10.1007/s11277-012-0674-7).

[11] Q. Liang, X. Wang, X. Tian, F. Wu, and Q. Zhang, "Two-dimensional route switching in cognitive radio networks: A gametheoretical framework", IEEE/ACM Trans. on Netw. TON, vol. 23, no. 4, pp. 1053-1066, 2015 (doi: 10.1109/TNET.2014.2315194). 
[12] Y. Liu, L. X. Cai, and X. S. Shen, "Spectrum-aware opportunistic routing in multi-hop cognitive radio networks", IEEE J. on Selec. Areas in Commun., vol. 30, no. 10, pp. 1958-1968, 2012 (doi: 10.1109/JSAC.2012.121111).

[13] A. Abbagnale and F. Cuomo, "Gymkhana: a connectivity-based routing scheme for cognitive radio ad hoc networks", in Proc. IEEE INFOCOM Conf. on Comp. Commun. Workshops, pp. 1-5, San Diego, CA, USA, 2010 (doi: 10.1109/INFCOMW.2010.5466618).

[14] Z. Yuan, J. B. Song, and Z. Han, "Interference aware routing using network formation game in cognitive radio mesh networks", IEEE J. on Selec. Areas in Commun., vol. 31, no. 11, pp. 2494-2503, 2013 (doi: 10.1109/JSAC.2013.131107).

[15] Q. Zhu, Z. Yuan, J. B. Song, Z. Han, and T. Basar, "Interference aware routing game for cognitive radio multi-hop networks", IEEE J. on Selec. Areas in Comm., vol. 30, no. 10, pp. 2006-2015, 2012 (doi: 10.1109/JSAC.2012.121115).

[16] C.-F. Shih, W. Liao, and H.-L. Chao, "Joint routing and spectrum allocation for multi-hop cognitive radio networks with route robustness consideration", IEEE Trans. on Wireless Commun., vol. 10, no. 9, pp. 2940-2949, 2011 (doi: 10.1109/TWC.2011.072011.101249).

[17] S. Kamruzzaman, E. Kim, D. G. Jeong, and W. S. Jeon, "Energyaware routing protocol for cognitive radio ad hoc networks", IET Commun., vol. 6, no. 14, pp. 2159-2168, 2012 (doi: 10.1049/iet-com.2011.0698).

[18] T. C. Clancy, "Achievable capacity under the interference temperature model", in Proc. IEEE INFOCOM Int. Conf. on Comp. Commun., pp. 794-802, Anchorage, AL, USA, 2007 (doi: 10.1109/INFCOM.2007.98).

[19] S. Touati, H. Boujemaa, and N. Abed, "Outage probability analysis of optimal and suboptimal interference aware routing protocols for multihop underlay cognitive radio networks", Trans. on Emerg. Telecommun. Technol., vol. 25, no. 7, pp. 736-744, 2014 (doi: 10.1109/INFCOM.2007.98).

[20] S. A. Doomari, G. Mirjalily, and J. Abouei, "Distributed game theory-based routing protocol for Cognitive Radio ad hoc networks", in Proc. Int. Joint Conf. on Comp. Sc. and Softw. Engin. JCSSE, Hatyai, Thailand, 2015, pp. 201-206 (doi: 10.1109/JCSSE.2015.7219796).

[21] C. E. Shannon, "A mathematical theory of communication", $A C M$ SIGMOBILE Mob. Comp. and Commun. Rev., vol. 5, no. 1, pp. 3-55, 2001 (doi: 10.1145/584091.584093).

[22] W. Saad, Z. Han, T. Basar, M. Debbah, and A. Hjorungnes, "Network formation games among relay stations in next generation wireless networks", IEEE Trans. on Commun., vol. 59, no. 9 , pp. 2528-2542, 2011 (doi: 10.1109/TCOMM.2011.062311.100046).

[23] J. Coimbra, G. Schütz, and N. Correia, "Energy efficient routing algorithm for fiber-wireless access networks: A network formation game approach", Comp. Netw., vol. 60, pp. 201-216, 2014 (doi: 10.1016/j.bjp.2013.11.014).

[24] H. Khanmirza and N. Yazdani, "Strategic network formation game for energy consumption balancing", Wireless Person. Commun., vol. 84, no. 2, pp. 841-865, 2015 (doi: 10.1007/s11277-015-2664-z).

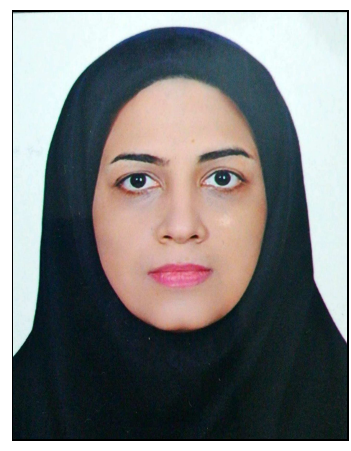

Soodeh Amiri-Doomari received her B.Sc. degree in Electrical Engineering and her M.Sc. degree in Telecommunication in 2011 from Kerman University and Isfahan University of Technology (IUT) respectively. She is currently a Ph.D. candidate in the department of Electrical Engineering, Yazd University. Her research interests are in the area of wireless ad-hoc networks, sensor networks, cognitive radio, game theory and mobile cognitive radio.

E-mail: s.amiridoomari@stu.yazd.ac.ir

Department of Electrical Engineering

Yazd University

Daneshgah Blvd.

89195-741 Yazd, Iran

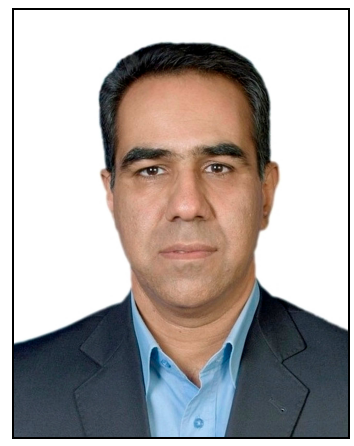

Ghasem Mirjalily received his Ph.D. degree in telecommunication engineering from Tarbiat Modarres University, Iran, in 2000. He was a visiting researcher at McMaster University, Canada, in 1998. Since 2000, he has been with Yazd University, Iran, where he is a full professor. Also, he is a senior member of IEEE. His interests include traffic engineering in wired and wireless networks.

E-mail: mirjalily@yazd.ac.ir

Department of Electrical Engineering

Yazd University

Daneshgah Blvd.

89195-741 Yazd, Iran

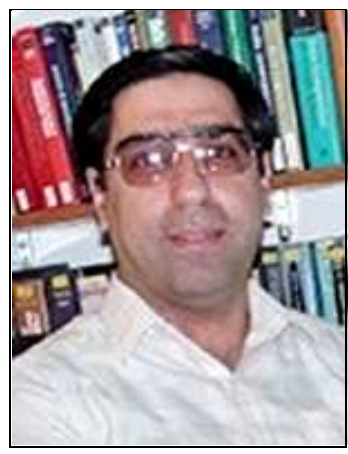

Jamshid Abouei received the B.Sc. degree in Electronics Engineering and the M.Sc. degree in Communication Systems Engineering both from the Isfahan University of Technology (IUT), Iran, in 1993 and 1996, respectively, and the Ph.D. degree in Electrical Engineering from the University of Waterloo in Waterloo, ON, Canada, in 2009. From 2009 to 2010, he was a Postdoctoral Fellow in the Multimedia Lab, in the Department of Electrical and Computer Engineering, at the University of Toronto, ON, Canada. Currently he is an Associate Professor in the Department of Electrical and Computer Engineering at the Yazd University. His research interests are in general areas of wireless ad hoc and sensor networks, with particular reference to energy efficiency and optimal resource allocations.

E-mail: abouei@yazd.ac.ir

Department of Electrical Engineering

Yazd University

Daneshgah Blvd.

89195-741 Yazd, Iran 